\title{
Reduced mechanical oscillations using the MAGLEV concept in vertical axis wind turbine
}

\begin{abstract}
Due to its low power design applications, the Vertical Axis Wind Turbine is more commonly employed for the standalone applications. The power generating capability in wind turbines is influenced by the mechanical dimensions of the blade including the shape of the blade and the angle of attack. The appropriate design of the blade shape and position tends to improvise the efficiency even at low wind speed. Initially the shape of the airfoil is designed and analyses and the position for a five blade structure is investigated. The degree of impact at angle of $30^{\circ}$ is found to have the highest lift coefficient for the chosen airfoil structure. The use of MAGLEV concept in the VAWT reduces the vibration by $37.5 \%$. Experimental results are presented with and without MAGLEV imported to the VAWT design. Also it is measured that the power generated with maglev system is increase by $12 \%$ compare to the normal wind turbine.
\end{abstract}

Keyword: VAWT; Maglev; Blade design; Blade position 